\title{
ENSAIOS NÃO DESTRUTIVOS PARA DIAGNÓSTICO DE ESTRUTURAS DE CONCRETO ARMADO: UMA REVISÃO SISTEMÁTICA
}

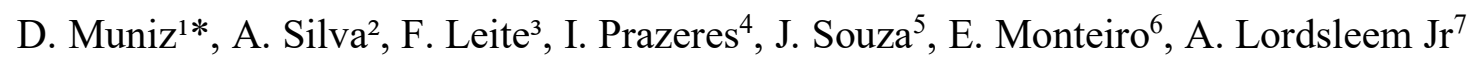 \\ *Autor de contato: dvm@poli.br \\ 1,2,3,4,5 Escola Politécnica de Pernambuco, Recife, Brasil.
}

\section{RESUMO}

O objetivo deste artigo consiste em apresentar uma revisão sistemática de estudos recentes sobre a aplicação de ensaios não destrutivos em estruturas de concreto armado. A metodologia utilizada seguiu as diretrizes do PRISMA (Preferred Reporting Items for Systematic Reviews and Meta Analysis), com triagem realizada no Portal de Periódicos da CAPES. Foram encontrados inicialmente 836 artigos e, a partir dos critérios de inclusão e exclusão, a seleção resultou em 24 artigos. A análise baseou-se nos ensaios utilizados e seus respectivos resultados, em função do tipo de dano avaliado. Observou-se as novas metodologias para detecção de danos, capazes de aumentar o grau de precisão e eficiência dos ensaios, as limitações dos ensaios e a integração entre dois ou mais métodos.

Palavras-chave: Ensaios não destrutivos; Concreto; Novas metodologias.

\begin{abstract}
The objective of this article is to present a systematic review of recent studies on the application of non-destructive tests on reinforced concrete structures. The methodology used followed the guidelines of PRISMA (Preferred Reporting Items for Systematic Reviews and Meta Analysis), with selection performed on the CAPES Journal Portal. Initially, 836 articles were found and, based on the inclusion and exclusion criteria, the selection resulted in 24 articles. The analysis was based on the tests used and their respective results, depending on the type of damage evaluated. It was observed the new methodologies for damage detection, able of increasing the degree of precision and efficiency of the tests, the limitations of the tests and the integration between two or more methods.
\end{abstract}

Key-words: Non-destructive testing, Concrete; New methodologies.

\section{INTRODUÇÃO}


A preocupação atual com a qualidade e o desempenho das construções tem contribuído para aumentar a relevância dos conceitos de vida útil dos materiais construtivos e de durabilidade, nas tomadas de decisões de projeto e execução das edificações. Tendo em vista que as exigências de qualidade estão se firmando, cada vez mais, no setor da construção civil, é de vital importância o desenvolvimento de alternativas que permitam, de forma eficaz e rápida, avaliar a qualidade das estruturas de concreto (Barreto et al., 2019).

O uso de métodos de ensaios não destrutivos (END) é uma maneira de viabilizar a inspeção e a avaliação do estado de conservação de construções civis, de forma econômica e eficiente. Dada sua natureza predominantemente não invasiva, os ensaios tipo END se constituem em uma das principais ferramentas de controle da qualidade de materiais e produtos, e já são amplamente utilizados em diversas atividades, tais como na indústria aeroespacial, no ramo automobilístico, no ramo elétrico e na construção naval (Cho, 2003).

Os ensaios não destrutivos permitem a coleta de informações como tamanho, profundidade, localização e estado da armadura, além de condições físicas e parâmetros que estão associados aos processos de deterioração ou risco de danos à estrutura, tudo isso causando pouco ou nenhum prejuízo ao elemento (Mapa da obra, 2016). Dentre os ensaios não destrutivos mais comumente aplicados estão: Termografia de Pulso Longo (LPT); Termografia infravermelha; Tomografia Ultrassônica (UT); Aplicação de sensores ultrassônicos e automotivos; Resistividade elétrica; Potencial Eletroquímico; Emissão Acústica (AE).

Existem normas específicas para cada método de ensaio, por isso é importante a correta qualificação do profissional que irá realizar o ensaio e a elaboração de procedimentos conforme tais normas. Existem também normas que regulam empresas de END, tais como a ISO 17020 (SGS, 2018).

Várias pesquisas são realizadas no intuito de ressaltar a aplicabilidade do ensaios não destrutivos. Neste sentido, este estudo teve como objetivo revisar a literatura relacionada aos ensaios não destrutivos mais utilizados em pesquisas recentes para avaliar as estruturas de concreto armado.

\section{PROCEDIMENTO}

\subsection{Estratégia de Pesquisa}

Esta revisão sistemática foi baseada nas diretrizes do modelo PRISMA, Preferred Reporting Items for Systematic Reviews and Meta-analyses (Liberati et al., 2009). Os artigos selecionados para esta revisão foram pesquisados no Portal de Periódicos da CAPES.

As palavras-chave utilizadas na busca foram "non-destructive testing", "ensaios não destrutivos", "concrete", "concreto", "inspection", "inspeção" combinadas com o booleano "AND" seguidas do booleano "NOT" para as palavras "mortar", "argamassa", "grout", "compressive".

\subsection{Critérios de inclusão}

Foram incluídos artigos em português e inglês, revisados por pares, sendo que para os artigos em português foram considerados os publicados nos 5 últimos anos e para os artigos em inglês foram considerados apenas os publicados nos 2 últimos anos, devido ao grande número de artigos encontrados quando a busca era ampliada para os últimos 5 anos.

\subsection{Critérios de exclusão}

Para os artigos em inglês foram excluídos os publicados em periódicos cujo qualis era inferior a A2, artigos repetidos para ambos os idiomas e que estavam inseridos em periódicos que não abordavam os ensaios não destrutivos em concreto dentro da engenharia civil.

\subsection{Seleção dos estudos}


Os artigos que restaram depois de aplicados os critérios já descritos, foram analisados com base nos títulos e resumos. Posteriormente, os artigos selecionados foram lidos por completo e selecionados de acordo com sua relevância dentro do tema proposto.

\section{RESULTADOS}

Utilizando a estratégia PRISMA de pesquisa, foram encontrados inicialmente 836 artigos e destes, 745 foram revisados por pares. Depois da aplicação dos critérios de inclusão, idioma inglês e português, publicados nos 2 últimos anos para os artigos em inglês e 5 últimos anos para os artigos em português, restaram 238 artigos. Posteriormente, os 238 artigos foram analisados por título e resumo, onde foram excluídos os artigos que não abordavam os ensaios não destrutivos dentro da engenharia civil, restando 50 artigos. Em seguida, os artigos foram avaliados por completo, analisando sua relevância dentro do tema proposto, o que resultou na exclusão de 26 artigos, ficando com um total final de 24 artigos para esta revisão bibliográfica, conforme ilustrado no Fluxograma 1, que contém todo o processo de seleção.
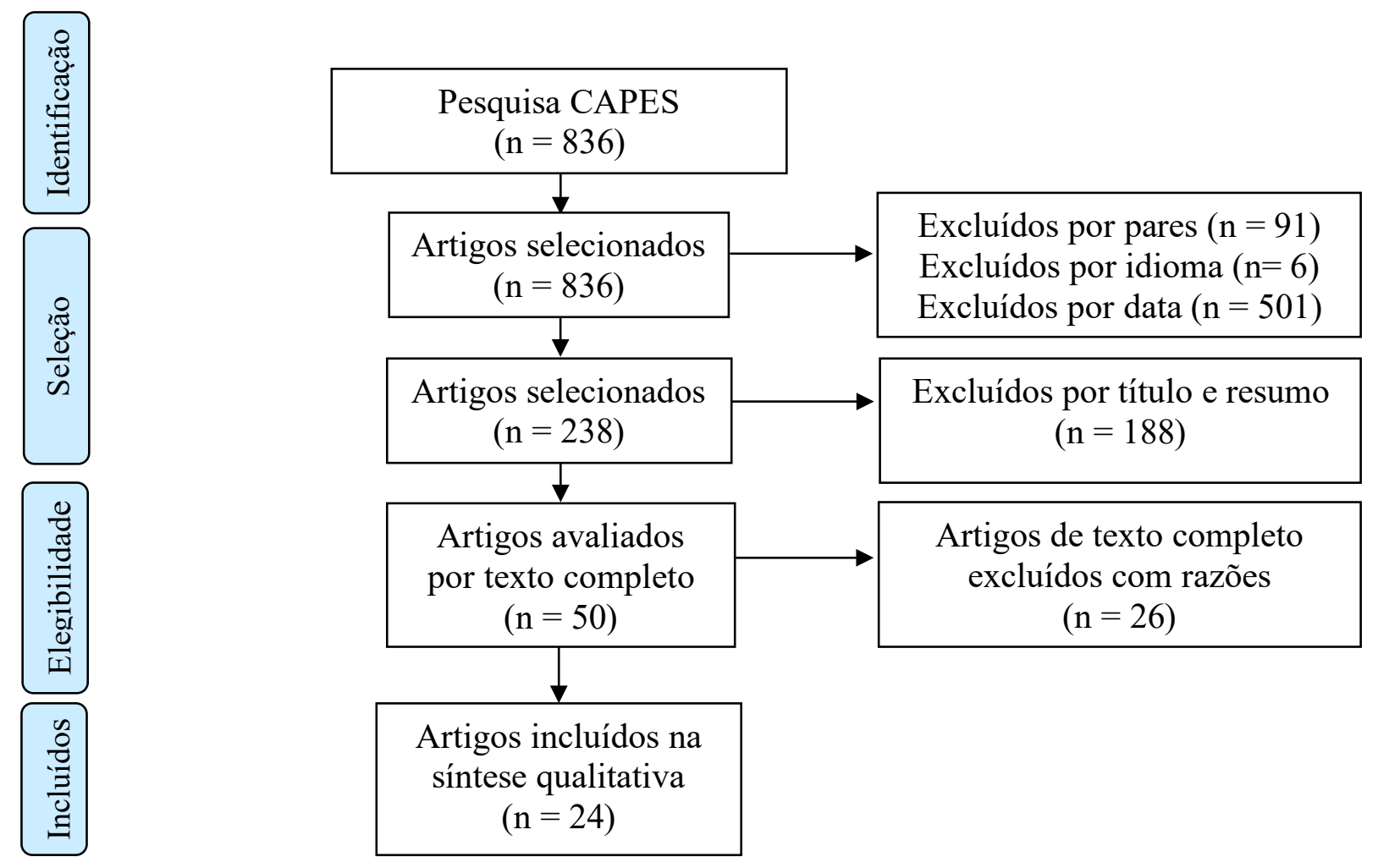

Fluxograma 1. Processo de seleção dos artigos Fonte: Elaborado pelos autores

A Tabela 1 apresenta um resumo de pontos importantes dos trabalhos selecionados, sendo eles: autor, ano, país, ensaio utilizado e defeito detectado. O estudo reuniu diferentes ensaios não destrutivos realizados em 11 países para detectar danos ou características do concreto e/ou armadura. Dessa forma, a revisão se propõe a apresentar os resultados obtidos por ensaios utilizados para cada tipo de manifestação patológica, bem como, discutir seus principais resultados e contribuições.

Tabela 1. Tabela resumo dos artigos selecionados 


\begin{tabular}{|c|c|c|c|c|c|}
\hline $\mathbf{N}^{\mathbf{0}}$ & Autor & Ano & País & Ensaio(s) & Defeito detectado \\
\hline 1 & Sun et al. & 2018 & China & $\begin{array}{l}\text { Excitação } \\
\text { eletromagnética aberta }\end{array}$ & Danos na armadura \\
\hline 2 & $\begin{array}{l}\text { Balamonica } \\
\text { et al. }\end{array}$ & 2020 & Índia & Sensor piezoelétrico & $\begin{array}{l}\text { Danos no concreto e medição } \\
\text { de deformações }\end{array}$ \\
\hline 3 & $\begin{array}{ll}\text { Song } & \text { e } \\
\text { Popovics } & \\
\end{array}$ & 2019 & EUA & Onda ultrassônica & $\begin{array}{l}\text { Fissuras próximas à superfície } \\
\text { do concreto }\end{array}$ \\
\hline 4 & Tran et al. & 2018 & $\begin{array}{l}\text { Coréia do } \\
\text { Sul }\end{array}$ & Termografia pulsada & $\begin{array}{l}\text { Delaminações com relação à } \\
\text { largura-profundidade }\end{array}$ \\
\hline 5 & $\begin{array}{l}\text { Lorenzi et } \\
\text { al. }\end{array}$ & 2016 & Brasil & $\begin{array}{l}\text { Velocidade de pulso } \\
\text { ultrassônico; } \\
\text { Termografia } \\
\text { Infravermelha; } \\
\text { Tomografia Ultrassônica }\end{array}$ & $\begin{array}{l}\text { Falhas de concretagem, } \\
\text { fissuras, heterogeneidades }\end{array}$ \\
\hline 6 & Teng et al. & 2019 & Suiça & $\begin{array}{l}\text { Teoria e aplicação de } \\
\text { microondas, com sensor } \\
\text { (permissividade) }\end{array}$ & $\begin{array}{l}\text { Teor de umidade } \\
\text { deterioração em concreto }\end{array}$ \\
\hline 7 & Zhou et al. & 2018 & China & $\begin{array}{l}\text { Radar de penetração no } \\
\text { solo (GPR) e métodos de } \\
\text { indução eletromagnética } \\
\text { (EMI) }\end{array}$ & $\begin{array}{l}\text { Diâmetro do vergalhão e a } \\
\text { espessura do cobrimento com } \\
\text { precisão por uma única } \\
\text { varredura }\end{array}$ \\
\hline 8 & $\begin{array}{l}\text { Ullah, } \\
\text { Jeong e Lee }\end{array}$ & 2018 & Suiça & $\begin{array}{lcc}\text { Aplicação de } & \text { sensor e a } \\
\text { utilização } & \text { de } & \text { um } \\
\text { aplicativo } & & \\
\end{array}$ & Avaliar integridade de barras. \\
\hline 9 & Ye et al. & 2018 & Japão & $\begin{array}{l}\text { Interpretação de dados } \\
\text { sonoros com utilização } \\
\text { de martelo e microfone }\end{array}$ & $\begin{array}{l}\text { Avaliação de estruturas de } \\
\text { concreto }\end{array}$ \\
\hline 10 & $\begin{array}{l}\text { Louhi } \\
\text { Kasahara et } \\
\text { al. }\end{array}$ & 2020 & Japão & $\begin{array}{l}\text { Martelamento } \\
\text { informações visuais. }\end{array}$ & Delaminações e vazios \\
\hline 11 & $\begin{array}{l}\text { Chakraborty } \\
\text { et al. }\end{array}$ & 2019 & Polônia & Sensores ultrassônicos & Fissuras \\
\hline 12 & $\begin{array}{l}\text { Fujino e } \\
\text { Siringoringo }\end{array}$ & 2020 & Japão & $\begin{array}{l}\text { Indução eletromagnética } \\
\text { de baixa frequência }\end{array}$ & $\begin{array}{l}\text { Avaliação geral das condiçõe } \\
\text { de estruturas }\end{array}$ \\
\hline 13 & $\begin{array}{l}\text { La Malfa } \\
\text { Ribolla et } \\
\text { al. }\end{array}$ & 2018 & Itália & Sensores ultrassônicos & $\begin{array}{l}\text { Danos concretos reforçados } \\
\text { com fibras }\end{array}$ \\
\hline 14 & $\begin{array}{l}\text { Słonski et } \\
\text { al. }\end{array}$ & 2020 & Polônia & $\begin{array}{l}\text { Tomografia ultrassônica } \\
\text { (UT) e Rede neural } \\
\text { convolucional (CNNs) }\end{array}$ & $\begin{array}{l}\text { Detecção automática de falhas } \\
\text { em elementos de concreto }\end{array}$ \\
\hline 15 & Rucka et al. & 2020 & Polônia & $\begin{array}{l}\text { Teste Ultrassônico (UT) } \\
\text { e Radar de penetração } \\
\text { no solo (GPR) }\end{array}$ & Detecção de lacunas de ar \\
\hline 16 & Zhang et al. & 2018 & França & Sensores ultrassônicos & Fissuras \\
\hline $\mathbf{N}^{0}$ & Autor & Ano & País & Ensaio(s) & Defeito detectado \\
\hline
\end{tabular}




\begin{tabular}{|c|c|c|c|c|c|}
\hline 17 & $\begin{array}{l}\text { Hashimoto } \\
\text { et al. }\end{array}$ & 2020 & Japão & $\begin{array}{l}\text { 3D SIBIE - Imagens de } \\
\text { pilha de amplitudes } \\
\text { espectrais com base no } \\
\text { eco de impacto }\end{array}$ & $\begin{array}{l}\text { Detecção de fissuras e vazios } \\
\text { no concreto. }\end{array}$ \\
\hline 18 & Sofi et al. & 2019 & Austrália & $\begin{array}{l}\text { Medição de cobrimento } \\
\text { e disposição de } \\
\text { armadura (Profometer); } \\
\text { Ensaio } \\
\text { permeabilidade ao ar } \\
\text { (Torrent); Resistividade } \\
\text { elétrica } \\
\text { Potencial eletroquímico } \\
(\text { Canin+) }\end{array}$ & $\begin{array}{l}\text { Avaliação geral das condições } \\
\text { de estruturas de concreto }\end{array}$ \\
\hline 19 & $\begin{array}{l}\text { Vashpanov } \\
\text { et al. }\end{array}$ & 2019 & $\begin{array}{l}\text { República } \\
\text { da Coréia }\end{array}$ & $\begin{array}{l}\text { 8-bit RGB (red, green } \\
\text { and blue) imagem }\end{array}$ & $\begin{array}{l}\text { Determinação de parâmetros } \\
\text { geométricos de fissuras em } \\
\text { concreto }\end{array}$ \\
\hline 20 & $\mathrm{Wu}$ et al. & 2018 & China & $\begin{array}{l}\text { Termografia } \\
\text { infravermelha }\end{array}$ & $\begin{array}{l}\text { Detecção de danos de impacto } \\
\text { de uma viga de PVA-ECC }\end{array}$ \\
\hline 21 & Pan et al. & 2018 & China & $\begin{array}{l}\text { Novo método baseado } \\
\text { em um sistema de } \\
\text { detecção de temperatura } \\
\text { distribuída }\end{array}$ & $\begin{array}{l}\text { Detecção de defeitos em } \\
\text { pontes tubulares de aço } \\
\text { preenchidas com concreto } \\
\text { (CFST) }\end{array}$ \\
\hline 22 & Huh et al. & 2018 & $\begin{array}{l}\text { Coréia do } \\
\text { Sul }\end{array}$ & $\begin{array}{l}\text { Termografia de Pulso } \\
\text { Longo (LPT) }\end{array}$ & $\begin{array}{l}\text { Efeito da profundidade, tempo } \\
\text { de aquecimento e vergalhões } \\
\text { sobre a capacidade de } \\
\text { detecção de delaminação }\end{array}$ \\
\hline 23 & $\begin{array}{l}\text { Dorafshan et } \\
\text { al. }\end{array}$ & 2018 & USA & $\begin{array}{l}\text { Detectores de borda } \\
\text { comuns e redes neurais } \\
\text { convolucionais } \\
\text { profundas (DCNN) }\end{array}$ & Detecção de fissuras \\
\hline 2 & $\begin{array}{l}\text { Takami } \\
\text { al }\end{array}$ & 2018 & Japão & Emissão acústica (AE) & Rachaduras em pontes \\
\hline
\end{tabular}

Fonte: Elaborada pelos autores

\section{DISCUSSÃO}

Os estudos foram classificados em função das manifestações patológicas abordadas, de forma que, dos 24 artigos selecionados, 5 artigos abordavam sobre cobrimento e/ou corrosão, 10 sobre fissuras e 9 sobre integridade estrutural.

\subsection{Cobrimento de armaduras}

A Tabela 2 apresenta os autores, países, ensaios executados e resultados encontrados dos artigos que discutiram sobre cobrimento de armaduras.

Tabela 2. Tabela resumo dos artigos com abordagem sobre cobrimento de armaduras 


\begin{tabular}{|c|c|c|}
\hline Autor (ano) & Ensaios utilizados & Resultados \\
\hline Huh et al. (2018) & $\begin{array}{l}\text { Termografia de } \quad \text { Pulso } \\
\text { Longo (LPT) }\end{array}$ & $\begin{array}{l}\text { Variação de dissipação de temperatura com } \\
\text { impactos de cobrimento que aumentam em } \\
\text { função do espaço dissipado. }\end{array}$ \\
\hline Tran et al. (2018) & $\begin{array}{lll}\text { Termografia } & \text { de } & \text { Pulso } \\
\text { Longo (LPT) } & & \end{array}$ & Difusividade térmica de $1,076 \mathrm{~cm}^{2} / \mathrm{min}$ \\
\hline Sofi et al. (2019) & $\begin{array}{l}\text { Medição de cobrimento e } \\
\text { disposição de armadura } \\
\text { (Profometer) e ensaio de } \\
\text { permeabilidade ao ar } \\
\text { (método Torrent) }\end{array}$ & $\begin{array}{l}\text { Os resultados mostraram que o cobrimento de } \\
\text { concreto, pode ser medido com uma precisão de } \\
\text { cerca de } \pm 1 \mathrm{~mm} \text {. }\end{array}$ \\
\hline Zhou et al. (2018) & $\begin{array}{l}\text { Radar de penetração no } \\
\text { solo (GPR) e métodos de } \\
\text { indução eletromagnética } \\
\text { (EMI) }\end{array}$ & $\begin{array}{l}\text { Conclui-se que o sensor duplo GPR-EMI } \\
\text { desenvolvido e o algoritmo proposto podem } \\
\text { estimar o diâmetro do vergalhão e a espessura do } \\
\text { cobrimento com precisão por uma única } \\
\text { varredura. }\end{array}$ \\
\hline $\begin{array}{l}\text { Fujino e } \\
\text { Siringoringo } \\
\quad(2020)\end{array}$ & $\begin{array}{l}\text { Indução eletromagnética } \\
\text { de baixa frequência }\end{array}$ & $\begin{array}{l}\text { A precisão do sistema eletromagnético comercial } \\
\text { para encontrar a profundidade da barra pode não } \\
\text { ser adequado. }\end{array}$ \\
\hline
\end{tabular}

Fonte: Elaborada pelos autores

A termografia infravermelha tem sido um dos ensaios não destrutivos mais utilizados, devido à facilidade de se obter o resultado e pela sua metodologia executiva. De acordo com o critério da Relação Sinal Ruído (RSR), utilizado por Huh, J. et al. (2018) é possível identificar a espessura do cobrimento com $7 \mathrm{~cm}$ em estruturas de concreto, entretanto, à medida que os cobrimentos aumentam, a eficácia do RSR reduz consideravelmente.

A limitação de alcance de acordo com a profundidade também foi apresentada por Tran et al. (2018), que determinou coeficientes de proporcionalidade entre tamanho e profundidade, chamado de relação largura-profundidade. Os autores analisaram o aumento da temperatura e os impactos de difusão gerados pelo experimento, concluindo que a delaminação, para uma relação largura-profundidade igual ou superior a 1,25, pode ser detectada usando uma câmera infravermelha de longo comprimento de onda adequada.

Sofi, M. et al. (2019), com o objetivo de detectar o cobrimento do concreto, utilizando a medição de cobrimento e disposição de armadura (Profometer) e ensaio de permeabilidade ao ar (método Torrent), mostraram que a corrosão aumenta a permeabilidade do ar e, consequentemente, reduz a qualidade da cobertura de concreto.

Zhou et al. (2018) utilizaram de forma integrada os ensaios de radar de penetração no solo (GPR) e método de indução eletromagnética (EMI), como métodos simultâneos de estimativa do diâmetro do vergalhão e espessura do cobrimento. Um experimento de laboratório apresentou erro máximo de estimativa para a espessura do cobrimento de $6,7 \%$. Um ensaio de campo foi realizado em um edifício recém-construído e apresentou erro de estimativa da espessura de cobrimento inferior a 5\%. O estudo conclui que o sensor duplo GPR-EMI desenvolvido e o algoritmo proposto podem estimar o diâmetro do vergalhão e a espessura do cobrimento com precisão por uma única varredura.

Fujino e Siringoringo (2020) relataram um método que utiliza indução eletromagnética de baixa frequência, por meio do qual os resultados mostraram que a precisão do sistema eletromagnético comercial para encontrar a profundidade da barra pode não ser adequado. 


\subsection{Corrosão}

A Tabela 3 apresenta os autores, países, ensaios executados e resultados encontrados dos artigos que discutiram sobre corrosão de armaduras.

Tabela 3. Tabela resumo dos artigos com abordagem sobre corrosão

\begin{tabular}{|c|c|c|}
\hline Auto & Ensaios utilizados & Resultados \\
\hline $\begin{array}{l}\text { Sofi et al. } \\
\text { (2019) }\end{array}$ & $\begin{array}{l}\text { Ensaio de permeabilidade } \\
\text { ao ar (método }\end{array}$ & $\begin{array}{l}\text { O estudo comprovou que uma determinada } \\
\text { quantidade de } \mathrm{NaCl} \text { diminui a permeabilidade do } \\
\text { ar no concreto, porém aumenta a corrosão. A } \\
\text { introdução de cinzas volantes apresentou como } \\
\text { resultado a redução da taxa de corrosão, mas com } \\
\text { pequeno efeito benéfico. }\end{array}$ \\
\hline $\begin{array}{c}\text { Fujino e } \\
\text { Siringoringo } \\
(2020)\end{array}$ & $\begin{array}{l}\text { Indução eletromagnética } \\
\text { de baixa frequência; } \\
\text { espectroscopia de perda de } \\
\text { energia de elétrons; } \\
\text { medições magnéticas } \\
\text { altamente sensíveis }\end{array}$ & $\begin{array}{l}\text { A precisão do sistema eletromagnético comercial } \\
\text { para encontrar a profundidade da barra pode não } \\
\text { ser adequado. Concluiu-se que o aço de cromo tem } \\
\text { resistência à corrosão significativamente maior do } \\
\text { que aço de carbono em concreto. Os resultados } \\
\text { mostraram a capacidade de medir mudanças de } \\
\text { espessura e verificar a presença de corrosão. }\end{array}$ \\
\hline
\end{tabular}

Fonte: Elaborada pelos autores

Sofi et al. (2019), com o objetivo de detectar a corrosão em elementos de concreto, utilizaram medições de potencial de meia célula com Canin +, para o mapeamento do estado de corrosão dos vergalhões, e medição de resistividade elétrica com Resipop, para obter a taxa de corrosão.

Os resultados mostraram que o acréscimo de uma pequena quantidade de $\mathrm{NaCl}$ aumentou a durabilidade do concreto, pois diminuiu a permeabilidade do ar em relação ao cobrimento, porém aumentou a corrosão. Além disso, a introdução de cinzas volantes parece ter resultado na redução da taxa de corrosão, entretanto o efeito benéfico foi pequeno.

Fujino e Siringoringo (2020) apresentaram três maneiras diferentes de avaliar corrosão. A primeira utilizou a indução eletromagnética de baixa frequência e seus resultados mostraram que a precisão do sistema eletromagnético comercial para encontrar a profundidade da barra pode não ser adequado, o que torna difícil o uso para avaliação de redução do diâmetro do vergalhão de aço devido à corrosão. A segunda utilizou espectroscopia de perda de energia de elétrons, com o objetivo de encontrar relação entre a alta resistência à corrosão e a formação de ferrugem para o rolamento aço de cromo em comparação com o aço carbono em concreto exposto ao ambiente costeiro. Nesta análise, foi possível concluir que o aço de cromo tem resistência à corrosão significativamente maior do que aço de carbono em concreto. O terceiro método, teste de corrente de frequência extremamente baixa para avaliação de defeitos mais profundos e extensos na infraestrutura do aço, apresentou resultados que mostraram a capacidade de medir mudanças de espessura e verificar a presença de corrosão.

\subsection{Fissuras}

A Tabela 4 apresenta os autores, países, ensaios executados e resultados encontrados dos artigos que discutiram sobre fissuras. 
Tabela 4 .Tabela resumo dos artigos com abordagem sobre fissuras

\begin{tabular}{|c|c|c|}
\hline Autor (ano) & Ensaios utilizados & Resultados \\
\hline $\begin{array}{l}\text { Song e } \\
\text { Popovics } \\
(2019)\end{array}$ & Onda ultrassônica & $\begin{array}{l}\text { Variação de ondas ultrassônicas em regiões } \\
\text { fissuradas. Fator F - K de caracterização de tipo de } \\
\text { dano definido. Localização onde o dano foi gerado. }\end{array}$ \\
\hline $\begin{array}{l}\text { Chakraborty } \\
\text { et al. }(2019)\end{array}$ & Onda ultrassônica & $\begin{array}{l}\text { Detecção eficiente de microfissuras com avanço de } \\
\text { propagação e aumento. }\end{array}$ \\
\hline $\begin{array}{l}\text { Zhang et al. } \\
\qquad(2018)\end{array}$ & Onda ultrassônica & $\begin{array}{l}\text { A alta sensibilidade do Locadiff permite a } \\
\text { caracterização de trincas com solicitações muito } \\
\text { limitadas. }\end{array}$ \\
\hline $\begin{array}{l}\text { La Malfa } \\
\text { Ribolla et } \\
\text { al. (2018) } \\
\end{array}$ & Onda ultrassônica & $\begin{array}{l}\text { Resultados eficientes sobre desempenho de colagem } \\
\text { de reforço de fibra de carbono em estrutura de } \\
\text { concreto, através de sensibilidade de sensor. }\end{array}$ \\
\hline $\begin{array}{l}\text { Pan et al. } \\
\text { (2018) }\end{array}$ & $\begin{array}{l}\text { Método baseado em um } \\
\text { sistema de detecção de } \\
\text { temperatura distribuída }\end{array}$ & $\begin{array}{l}\text { Variação térmica encontrada de acordo com vazios } \\
\text { entre interface da estrutura de estudo. }\end{array}$ \\
\hline $\begin{array}{l}\text { Hashimoto } \\
\text { et al. }(2020)\end{array}$ & Emissão acústica (EA) & $\begin{array}{l}\text { Estimaram e exibiram danos internos e informações } \\
\text { geométricas dos materiais que constituem as } \\
\text { camadas de concreto, como pavimento asfáltico e } \\
\text { revestimento reforçado com fibra. }\end{array}$ \\
\hline $\begin{array}{l}\text { Takamine } \\
\text { et al (2018) }\end{array}$ & Emissão acústica (EA) & $\begin{array}{l}\text { Diagnóstico de fissuras in loco e padronização da } \\
\text { caracterização em laboratório. }\end{array}$ \\
\hline $\begin{array}{l}\text { Dorafshan et } \\
\text { al. (2018) }\end{array}$ & $\begin{array}{l}\text { DCNN e detecção de bordas } \\
\text { através de imagens }\end{array}$ & $\begin{array}{l}\text { Detecção dificuldades de padronização pelo método } \\
\text { DCNN e precisão favorável para o método de } \\
\text { detecção de bordas. }\end{array}$ \\
\hline $\begin{array}{l}\text { Vashpanov } \\
\text { et al. } \\
\text { (2019) }\end{array}$ & $\begin{array}{l}\text { DCNN e detecção de bordas } \\
\text { através de imagens }\end{array}$ & $\begin{array}{l}\text { Detecção de fissuras com imagens, porém com } \\
\text { limitações. A precisão dos parâmetros estimados } \\
\text { obtidos com a distribuição não pode ser ultrapassada } \\
\pm 10 \% \text { para a largura e } \pm 15 \% \text { para a profundidade } \\
\text { no ambiente de laboratório. }\end{array}$ \\
\hline $\begin{array}{l}\text { Sun et al. } \\
\quad(2018)\end{array}$ & $\begin{array}{l}\text { Simulações tridimensionais } \\
\text { de elementos finitos e } \\
\text { experimentos comparando o } \\
\text { efeito de magnetização }\end{array}$ & $\begin{array}{l}\text { A técnica eletromagnética aberta tem um efeito de } \\
\text { magnetização semelhante e uma boa capacidade de } \\
\text { detecção de defeitos em comparação com as bobinas } \\
\text { tubulares tradicionais. Uma nova técnica MFL SHM } \\
\text { elétrica para vergalhões de concreto usando bobinas } \\
\text { eletromagnéticas abertas como magnetizadores. }\end{array}$ \\
\hline $\begin{array}{l}\text { Fujino e } \\
\text { Siringoringo } \\
\quad(2020)\end{array}$ & $\begin{array}{l}\text { Algoritmo em sistema } \\
\text { montado em veículo em } \\
\text { movimento. Método de } \\
\text { previsão de falhas usando a } \\
\text { simulação de tempo de vida } \\
\text { e dados de inspeção do local. }\end{array}$ & $\begin{array}{l}\text { Esse sistema foi capaz de identificar as fissuras e } \\
\text { suas dimensões. O método de assimilação proposto } \\
\text { reproduz as rachaduras internas mais prováveis sobre } \\
\text { o volume de domínios de análise com sucesso. }\end{array}$ \\
\hline
\end{tabular}

Fonte: Elaborada pelos autores

A propagação de ondas para identificação de fissuras foi utilizada por Song e Popovics (2019), onde com o auxílio de um aparelho ultrassônico foi possível detectar os danos induzidos através da propagação de ondas nessas fissuras, gerando amplitude de reprodução das ondas com as 
fendas oriundas dos danos. Os autores reforçaram que os danos gerados artificialmente diferem dos danos causados nas estruturas naturalmente, variando de acordo com diversos fatores, como a morfologia do dano, conectividade geométrica e dimensões.

Segundo Chakraborty et al. (2019), os sensores ultrassônicos de técnica difusa obtiveram resultados mais sensíveis detectados internamente e o recurso de detecção mostrou-se eficiente também para a localização da fissura gerada. Ainda segundo os autores, a amplitude pico a pico e $\mathrm{o}$ indicador do coeficiente tiveram um destaque na análise, pois ambos evoluem continuamente e seguem bem as três fases que descrevem os mecanismos de falha: o início das microfissuras, a propagação das rachaduras e a falha final.

Sobre a sensibilidade mencionada por Chakraborty et al. (2019), esta foi mensurada por Zhang et al. (2018) que, simulando uma mudança de pressão menor que $1 \mathrm{kPa}$, detectou mudanças estruturais (microdeformações) nas fissuras, possíveis de visualizar através dos ultrassons difusos.

Como método de avaliação La Malfa, Ribolla et al. (2018) buscaram analisar o desempenho de colagem do reforço de fibra de carbono em estruturas de concreto. A análise foi modelada numericamente usando um código comercial de elementos finitos e validada experimentalmente por meio de testes de amostras de concreto reforçadas com tiras de CFRP (Compósitos Reforçados com Fibras de Carbono). Os autores utilizaram também sensores ultrassônicos para desenvolver o estudo, apresentando uma metodologia mais eficiente e sensível do que as convencionais, pico a pico e RMS (Raiz do valor quadrático médio).

O estudo de Pan et al. (2018) foi realizado em estruturas tubulares preenchidas de concreto, utilizadas para construção de pontes e viadutos, chamadas pontes arcos, onde a principal preocupação foi a interface entre os materiais (o aço e o concreto preenchido). Os autores apresentaram os resultados experimentais utilizando a variação de temperatura para mapear possíveis alterações de espaçamento entre os elementos. No caso dos vazios maiores, a temperatura se dissipava com maior variação entre elas. Para um único modelo, a temperatura da altura máxima do vazio foi a mais alta, a taxa de aumento foi a mais rápida e a taxa de resfriamento foi a mais lenta (Pan et al. 2018).

A variação de temperatura para o modelo com diferentes formas de vazio, apresentou que quanto maior o vazio, maior a temperatura, pois, devido a existência do vazio, o calor absorvido pela superfície superior é transferido lentamente para a área do vazio e então acumulado (Pan et al. 2018).

Takamine et al. (2018) realizaram ensaios com sistema de monitoramento de emissão acústica (EA) com sensores piezoelétricos. O estudo foi desenvolvido in loco em estruturas de pontes com uma metodologia baseada em ondas elásticas geradas pelo fraturamento do material e captada por estes sensores.

Eco de impacto é um método acústico de avaliação de estruturas de concreto, no qual se baseia no uso de ondas elásticas geradas por impacto que se propagam através do concreto e são prospectadas por falhas internas ou superfícies externas. Vazios no concreto, delaminações, rachaduras e localização de falhas também podem ser identificadas com o método. (HASHIMOTO et al. 2020).

Hashimoto et al. (2020), desenvolveu uma melhoria para o procedimento SIBIE (imagem de pilha de amplitudes espectrais com base no eco de impacto), de forma que neste estudo, as etapas de medição e computação foram estendidas para o modelo tridimensional (3D), usando uma unidade de matriz multisensor (acelerômetro) para medição no local, onde o processo de cálculo permitiu estimar e exibir os danos internos e informações geométricas dos materiais que constituem as camadas de concreto, como pavimentos asfálticos e revestimentos reforçados com fibra. 
A detecção de fissuras em estruturas de concreto também pode ser identificada por outras metodologias, além da emissão de ondas ultrassônicas e eco impacto, como no caso de Dorafshan et al. (2018) e Vashpanovet al. (2019) que determinaram a inspeção por detecção de bordas e DCNN (Redes neurais convolucionais profundas), algoritmos para detecção de fissuras em concreto com base em imagens.

Entretanto, de acordo com Dorafshan et al. (2018), a padronização de algoritmos para determinadas imagens nos conjuntos de dados estudados reduz a precisão em casos de variações de cada situação, como em condições de baixa iluminação, câmeras com resolução inferior, e presença de sombras.

Os autores ainda realizaram um comparativo entre as DCNN e o método de detecção de bordas, destacando que os esquemas de detecção de borda obtiveram resultados razoavelmente positivos. Com melhor desempenho, o método detectou com precisão cerca de $79 \%$ dos pixels fissurados e obteve excelentes resultados na detecção de rachaduras maiores que $0,1 \mathrm{~mm}$.

De acordo com Fujino e Siringoringo (2020) dois métodos são usados para detectar fissuras. O primeiro método utiliza um algoritmo em um sistema que foi montado em um veículo em movimento. Esse sistema foi capaz de identificar as fissuras em duas dimensões. O segundo é um método de previsão de falhas usando a simulação de tempo de vida acoplada com dados de inspeção do local. O método de assimilação proposto reproduz as rachaduras internas mais prováveis sobre o volume de domínios de análise com sucesso.

\subsection{Integridade estrutural}

A Tabela 5 apresenta os autores, países, ensaios executados e resultados encontrados dos artigos que discutiram sobre integridade estrutural.

Tabela 5 .Tabela resumo dos artigos com abordagem sobre integridade estrutural

\begin{tabular}{|c|c|c|}
\hline & & \\
\hline $\begin{array}{l}\text { Fujino e } \\
\text { Siringoringo } \\
(2020)\end{array}$ & $\begin{array}{l}\text { Veículo de inspeção com sistema } \\
\text { de mapeamento, sistema de } \\
\text { detecção com laser remoto de alta } \\
\text { velocidade, entre outros. }\end{array}$ & $\begin{array}{l}\text { O veículo de inspeção equipado com sistema } \\
\text { de mapeamento, sistema de deteç̧ão com } \\
\text { laser remoto e o TLS apresentaram } \\
\text { resultados positivos. }\end{array}$ \\
\hline $\begin{array}{l}\text { Balam } \\
\text { et al. }(\end{array}$ & $\begin{array}{l}\text { Investigação experimental com } \\
\text { unidades de sensor de titanato de } \\
\text { zirconato de chumbo inteligentes }\end{array}$ & \\
\hline & $\begin{array}{l}\text { Velocidade de pulso ultrassônico } \\
\text { (VPU); Termografia Infravermelha; } \\
\text { Tomografia Ultrassônica para } \\
\text { avaliar resistência/ densidade }\end{array}$ & $\begin{array}{l}\text { rego de ensaios não destrutivos } \\
\text { obter indicações importantes para a } \\
\text { zação do concreto e dados sobre a }\end{array}$ \\
\hline & $\begin{array}{lll}\text { Antena inteligente } & \text { (sensor } \\
\text { eletromagnético) }\end{array}$ & $\begin{array}{l}\text { or é capaz de detectar o teor de } \\
\text { e de forma não destrutiva. }\end{array}$ \\
\hline & $\begin{array}{l}\text { ção de dados sonoros com } \\
\text { de r martelo } \\
\text { dorizado) e microfone e } \\
\text { s de máquina avançada }\end{array}$ & $\begin{array}{l}\text { que o esquema } \\
\text { ação de precisão } \\
\text { ia e baixa carga }\end{array}$ \\
\hline $\begin{array}{l}\text { Louhi } \\
\text { Kasahara et } \\
\text { al.(2020) }\end{array}$ & $\begin{array}{l}\text { Martelamento } \\
\text { visuais }\end{array}$ & $\begin{array}{l}\text { cia da qualidade da supervisão } \\
\text { aumentada, resultando em um } \\
\text { ral no desempenho. }\end{array}$ \\
\hline & $\begin{array}{l}\text { Tomografia ultrassônica (UT) e } \\
\text { Rede neural convolucional (CNNs). }\end{array}$ & $\begin{array}{l}\text { A metodologia obteve resultados e } \\
\text { confirmam que podem ser úteis para detectar }\end{array}$ \\
\hline
\end{tabular}




\begin{tabular}{|c|c|c|}
\hline Autor (ano) & Ensaios utilizados & Resultados \\
\hline & $\begin{array}{l}\text { São realizadas varreduras } \\
\text { mostrando a parte interna do } \\
\text { membro de concreto investigado. }\end{array}$ & $\begin{array}{l}\text { automaticamente falhas em elementos de } \\
\text { concreto com altíssima precisão. }\end{array}$ \\
\hline $\begin{array}{l}\text { Rucka et al. } \\
\quad \text { l.(2020) }\end{array}$ & $\begin{array}{l}\text { Teste Ultrassônico (UT) e Radar de } \\
\text { penetração no solo (GPR) }\end{array}$ & $\begin{array}{l}\text { A inspeção integrada dos dois ensaios } \\
\text { pareceu ser eficaz para diagnósticos } \\
\text { estruturais subterrâneas em edifícios de } \\
\text { patrimônio cultural. }\end{array}$ \\
\hline $\begin{array}{l}\text { Wu et al. } \\
\text { (2018) }\end{array}$ & Termografia Infravermelha & $\begin{array}{l}\text { O método proposto é inovador e abordagem } \\
\text { é eficaz para detectar danos de impacto. }\end{array}$ \\
\hline $\begin{array}{l}\text { Ullah, Jeong } \\
\text { e Lee et al. } \\
\quad(2018)\end{array}$ & $\begin{array}{l}\text { Aplicação de sensor e a utilização } \\
\text { de um aplicativo, com o uso de } \\
\text { algoritmo de redução de } \\
\text { dimensionalidade de mapeamento } \\
\text { de característica isométrica e } \\
\text { florestas aleatórias. }\end{array}$ & $\begin{array}{l}\text { O método proposto pôde verificar barras } \\
\text { quebradas ou não de acordo com os dados } \\
\text { reais do sensor e foi capaz de prever } 97 \% \text { de } \\
\text { sinais corretos entre e sinais de crack em } \\
\text { dados de sensor não classificados. }\end{array}$ \\
\hline
\end{tabular}

Fonte: Elaborada pelos autores

Fujino e Siringoringo (2020) apresentaram um artigo com vários métodos para diagnóstico das estruturas, tais como: veículo de inspeção equipado com um sistema de mapeamento que detecta defeitos internos no revestimento de concreto e apresentou resultados encorajadores de aplicações de campo; sistema de deteç̧ão com laser remoto de alta velocidade, com base no método de martelamento que foi desenvolvido para detectar defeitos na parede de concreto de um túnel ferroviário que apresentou resultados idênticos aos medidos com método de martelagem; a digitalização a laser para obter medições 3D confiáveis de estruturas em grande escala e os resultados mostraram que o sistema de reconhecimento automático pode reconhecer com sucesso duas formas de membros de aço.

Os autores também apresentaram a metodologia do Terrestrial Laser Scanning (TLS) para levantamento utilizando um método de registro de nuvens de pontos de pontes escaneadas a laser que apresentou taxas de sucesso do bruto e o registro foi de $100 \%$ e dois métodos com veículos aéreos não tripulados (UAV). O primeiro, composto por UAV com concha esférica de rotação passiva, permitiu uma visão aérea completa sob a ponte, tornando possível capturar partes críticas da ponte onde os danos ocorrem com frequência. $\mathrm{O}$ segundo (multicóptero de duas rodas com tecnologia de modelagem tridimensional), que demonstra a utilização de UAV para inspeção de pontes, permitiu verificar condições da ponte, possíveis danos e defeitos.

Já Balamonica et al. (2020) apresentaram um método para detectar danos estruturais utilizando técnicas não tripuladas de monitoramento de integridade estrutural e sensores feitos de materiais de titanato de zirconato de chumbo. Foi comprovado que o método é promissor para diminuir o volume de dados coletados dos sensores para identificar danos em uma estrutura.

$\mathrm{O}$ artigo apresentado por Lorenzi et al. (2016) evidenciou como poderiam ser empregados os ensaios não destrutivos para controle de estruturas através de 4 estudos de caso e concluíram que os ensaios não destrutivos são ferramentas úteis para a análise de estruturas, visto que os dados obtidos permitem obter indicações importantes para a caracterização do concreto, bem como dados sobre a homogeneidade e a qualidade da estrutura.

Teng et al. (2019) apresentaram uma técnica de detecção usando uma antena (sensor) inteligente para a avaliação não destrutiva do teor de umidade e inspeção de deterioração em blocos de concreto. O sensor é capaz de detectar o teor de umidade de forma não destrutiva e pode ser uma técnica potencial para manutenção e controle de qualidade do desempenho do edifício. 
No artigo desenvolvido por Ye et al. (2018) o objetivo foi construir um sistema de análise de resposta à marteladas no concreto usando aprendizado de máquina online, que é capaz de imitar habilidade de especialista humano em martelar investigação de resposta em estruturas de concreto. Os resultados demonstraram que o esquema proposto obteve uma avaliação de precisão favorável com alta eficiência e baixa carga de computação.

Segundo Louhi Kasahara et al. (2020), uma abordagem ativa fracamente supervisionada e reforçada por informação visual para a inspeção de estruturas de concreto usando métodos acústicos foi utilizada para detecção de defeitos em estruturas de concreto. Experiências em condições de laboratório, utilizando blocos de teste de concreto com defeitos provocados de delaminações e vazios, e em condições de campo, utilizando túnel simulado e módulo de martelamento automatizado foi realizado. A consistência da qualidade da supervisão fraca foi aumentada, resultando em um aumento geral no desempenho.

Já os autores Słonski et al. (2020), utilizaram tomografia ultrassônica e uma rede neural convolucional para automatizar a avaliação de defeitos e falhas em elementos de concreto. A metodologia apresentada obteve resultados que confirmam que podem ser úteis para detectar automaticamente falhas em elementos de concreto com altíssima precisão.

Rucka et al. (2020) revelaram no artigo a detecção de lacunas de ar subterrâneas e outras anomalias como paredes de tijolos, canos, entulho e restos humanos, localizado sob os ladrilhos de pedra e as lápides, utilizando modelos de laboratório ilustrando o comportamento das ondas eletromagnéticas e ultrassônicas com teste ultrassônico integrado e do radar de penetração no solo. Os métodos se complementaram, permitindo a exclusão de suas limitações. A inspeção integrada das duas técnicas pareceram eficazes para diagnósticos não destrutivos.

No artigo desenvolvido por Wu et al. (2018) foi utilizada termografia infravermelha para detectar danos de impacto em uma estrutura de viga feita de um compósito cimentício reforçado com fibra de álcool polivinílico (PVA-ECC). Por meio de investigação experimental, os resultados demonstraram que diferentes graus de dano correspondem a diferentes características térmicas infravermelhas.

Já os autores Ullah, Jeong e Lee (2018) desenvolveram um aplicativo que classifica os sinais de campo magnético de vários canais, conforme medido a partir dos postes de concreto armado para monitoramento da integridade estrutural com o uso de algoritmo de redução de dimensionalidade de mapeamento de característica isométrica (ISOMAP) e florestas aleatórias. O aplicativo pôde perceber se as barras estavam quebradas ou não e foi capaz de prever $97 \%$ de sinais corretos entre e sinais de crack em dados de sensor não classificados.

\section{MANUTENÇÃO}

A ausência da realização de manutenções preventivas é um dos fatores capazes de afetar o processo de deterioração de estruturas de concreto armado. Por isso, é importante ressaltar a necessidade da execução de um programa de manutenção que contemple inspeções períodicas ao sistema estrutural, possibilitando a avaliação do estado de conservação da estrutura.

As técnicas de ensaios não destrutivos (END) se apresentam como uma alternativa eficiente para tais inspeções, visto que, por não alterarem as características da estrutura, podem ser realizadas durante a fase de uso das edificações. Assim, é possível que os problemas sejam detectados ainda no estágio inicial, evitando que as manifestações patológicas evoluam, aumentem os danos, os riscos e os custos da manutenção ou reparo.

Os artigos analisados apresentaram diversas metodologias de END capazes de coletar dados com qualidade e auxiliar na avaliação do estado de conservação das estruturas. Sabe-se que o programa de manutenção deve considerar as características e condições de exposição para cada 
tipo de estrutura de concreto, entretanto, com base nos resultados dos artigos analisados, sugerese um programa de manutenção genérico, disposto na Tabela 6 , como contribuição ao tema.

Tabela 6. Sugestão de programa de manutenção preventiva para estruturas de concreto armado

\begin{tabular}{|c|c|c|c|}
\hline Atividade & $\begin{array}{l}\text { Ensaios não destrutivos } \\
\text { sugeridos }\end{array}$ & Periodicidade & Responsável \\
\hline $\begin{array}{l}\text { Verificação e mapeamento } \\
\text { de fissuras, trincas ou } \\
\text { rachaduras }\end{array}$ & $\begin{array}{l}\text { Onda ultrassônica; Emissão } \\
\text { acústica; Detecção de } \\
\text { bordas e Redes neurais } \\
\text { convolucionais profundas }\end{array}$ & \multirow{4}{*}{ A cada 1 ano } & \multirow{4}{*}{$\begin{array}{lr}\text { Profissional } \\
\text { habilitado } \\
\text { experiência } & \text { com } \\
\text { patologia } & \text { das } \\
\text { estruturas } & \text { de } \\
\text { concreto. Deve } & \text { ser } \\
\text { elaborado um } \\
\text { relatório técnico } \\
\text { com emissão de } \\
\text { ART (Anotação de } \\
\text { Responsabilidade } \\
\text { Técnica) do serviço. }\end{array}$} \\
\hline $\begin{array}{l}\text { Verificação de manchas } \\
\text { superficiais }\end{array}$ & Termografia infravermelha & & \\
\hline $\begin{array}{l}\text { Verificação da presença } \\
\text { de corrosão das armaduras }\end{array}$ & $\begin{array}{l}\text { Radar de penetração no solo } \\
\text { e Métodos de Indução } \\
\text { eletromagnética }\end{array}$ & & \\
\hline $\begin{array}{l}\text { Verificar a integridade } \\
\text { estrutural }\end{array}$ & $\begin{array}{l}\text { Tomografia ultrassônica e } \\
\text { Rede neural convolucional }\end{array}$ & & \\
\hline
\end{tabular}

Fonte: Elaborada pelos autores

\section{CONCLUSÃO}

Com base nos artigos analisados, mediante uma revisão sistemática, foi possível observar que diversas tecnologias têm sido aplicadas em ensaios não destrutivos de estruturas de concreto armado, resultando na formulação de novos métodos avaliativos, capazes de aumentar o grau de precisão e eficiência quando comparados às metodologias tradicionais.

Os estudos abordaram métodos com resultados satisfatórios e grande potencial de detecção de danos, como a termografia infravermelha, os sensores ultrassônicos e a emissão acústica. Alguns conceitos inovadores foram utilizados, como as Redes Neurais Convolucionais Profundas (DCNN), que possibilitam a detecção de danos por meio de algoritmos e processamento de imagens.

Também foi possível observar contribuições quanto às limitações dos métodos, como no caso da precisão da Indução Eletromagnética de Baixa Frequência e da relação largura-profundidade da Termografia de Pulso Longo (LPT). Alguns ensaios foram realizados em estágios iniciais de investigação e apresentaram resultados promissores, como os sensores de materiais de titanato de zirconato de chumbo.

Além dos resultados individuais dos ensaios, alguns estudos apresentaram experimentos com a intregração de dois ou mais métodos, como a associação entre a Tomografia Ultrassônica com a Rede neural convolucional (DCNN) e a integração entre o Radar de Penetração no Solo (GPR) com o método de indução eletromagnética (EMI) e com o Teste Ultrassônico (UT). Neste último caso, os métodos se complementaram, permitindo inclusive a exclusão de suas limitações.

Conclui-se que, a aplicação de novas tecnologias nos ensaios não destrutivos, tem desenvolvido o aprimoramento das inspeções em estruturas de concreto. Sugere-se que estudos futuros sejam direcionados a aperfeiçoar e incrementar as metodologias abordadas, a fim de obter resultados com nível ainda maior de precisão e qualidade, contribuindo para as análises do estado de conservação das estruturas e seus diagnósticos. 


\section{REFERÊNCIAS}

BARRETO et al. Principais manifestações patológicas encontradas em edifícios históricos. JUNIOR, B.B.; RABBANI, E.R.; ZLATAR, T. [Orgs.]. Aplicações do método PRISMA para revisão sistemática da literatura na Engenharia Civil. Recife: EDUPE, p. 73, 2019. 252p.

CHAKRABORTY, J.; KATUNIN, A.; KLIKOWICZ, P.; SALAMAK, M. Early Crack Detection of Reinforced Concrete Structure Using Embedded Sensors. Sensors, v.19, 3879, 2019.

CHO, Y. S. Non-destructive Testing of High Strength Concrete using Spectral Analysis of Surface Waves. NDT\&E International. [s.l.], n. 36, p. 229-235, 2003.

DORAFSHAN, S.; THOMAS, R. J.; MAGUIRE, M. Comparison of deep convolutional neural networks and edge detectors for image-based crack detection in concrete. Construction and Building Materials, v. 186, p.1031-1045, 2018.

FUJINO, Y.; SIRINGORINGO, D. Recent research anddevelopment programs for infrastructures maintenance, renovation and management in Japan. Structure and Infrastructure Engineering, v.16, p. 3-25, 2020.

HASHIMOTO, K.; SHIOTANI, T.; OHTSU, M. Application of Impact-Echo Method to 3D SIBIE Procedure for Damage Detection in Concrete. Applied Sciences, v. 10, 2729, 2020.

HUH, J.; MAC, V.H.; TRAN, Q.H.; LEE, K.-Y.; LEE, J.-I.; KANG, C. Detectability of Delamination in Concrete Structure Using Active Infrared Thermography in Terms of Signal-toNoise Ratio. Applied. Sciences,v. 8, 1986, 2018.

JUNIOR, B.B.; RABBANI, E.R.; ZLATAR, T. [Orgs.]. Aplicações do método PRISMA para revisão sistemática da literatura na Engenharia Civil. Recife: EDUPE, p. 73, 2019. 252p.

K, B. et al. Piezoelectric sensor-based damage progression in concrete through serial/parallel multi-sensing technique', Structural Health Monitoring, v. 19, p. 339-356, 2020.

LIBERATI, A. et al. The PRISMA statement for reporting systematic reviews and meta-analyses of studies that evaluate health care interventions: explanation and elaboration. Journal of clinical epidemiology, v. 62, n. 10, p. e1-e34, 2009.

LORENZI, A et al. Emprego de Ensaios Não Destrutivos para Inspeção de Estruturas de Concreto. Revista de Engenharia Civil IMED, v. 3, n. 1, p. 3-13, 2016.

LOUHI KASAHARA, J.Y.; YAMASHITA, A.; ASAMA, H. Acoustic Inspection of Concrete Structures Using Active Weak Supervision and Visual Information. Sensors, v. 20, 629, 2020.

MAPA DA OBRA. Métodos não destrutivos auxiliam na identificação de manifestações patológicas em estruturas de concreto. 2016. Disponível em: https://www.mapadaobra.com.br/inovacao/veja-como-funciona-ensaio-de-esclerometria /.Acesso em: 24 ago. 2020.

PAN, S.; ZHU, Y.; LI, D.; MAO, J. Interface Separation Detection of Concrete-Filled Steel Tube Using a Distributed Temperature Measuring System. Applied. Sciences, v. 8, 1653, 2018.

RIBOLLA, E.L.M.; HAJIDEHI.M.R; RIZZO, P; SCIMEMI, G.F.; SPADA, A.; GIAMBANCO, G. Ultrasonic inspection for the detection of debonding in CFRP-reinforced concrete. Structure and Infrastructure Engineering, v.14, p. 807-816, 2017. 
RUCKA, M.; WOJTCZAK, E.; ZIELINSKA, M. Integrated Application of GPR and Ultrasonic Testing in the Diagnostics of a Historical Floor. Materials, v. 13, 2547, 2020.

SGS (2018). Ensaios Não destrutivos. O que é preciso saber? Uma discussão inicial sobre os ensaios não destrutivos e suas aplicações diretas. 2018. Disponível em: https://www.sgsgroup.com.br. Acesso em: 20 ago. 2020.

SLONSKI, M.; SCHABOWICZ, K.; KRAWCZYK, E. Detection of Flaws in Concrete Using Ultrasonic Tomography and Convolutional Neural Networks. Materials, v. 13, p. 1 - 16, 2020.

SOFI, M., OKTAVIANUS, Y., LUMANTARNA, E., RAJABIFARD, A., DUFFIELD, C., ; MEDIS, P. (2019). Condition assessment of concrete by hybrid non-destructive tests. Journal of Civil Structural Health Monitoring. v 9, p.339-351, 2019

SONG, H.; POPOVICS, J.S. Contactless Ultrasonic Wavefield Imaging to Visualize NearSurface Damage in Concrete Elements. Applied. Sciences, v. 9, 3005, 2019.

SUN, Y. et al. Magnetic flux leakage structural health monitoring of concrete rebar using an open electromagnetic excitation technique, Structural Health Monitoring, v. 17, p. 121-134, 2018

TAKAMINE, H.; WATABE, K.; MIYATA, H.; ASAUE, H.; NISHIDA, T.; SHIOTANI, T. Efficient damage inspection of deteriorated RC bridge deck with rain-induced elastic wave. Construction and Building Materials, v.162, p. 908-913, 2018

TENG, K.H.; KOT, P.; MURADOV, M.; SHAW, A.; HASHIM, K.; GKANTOU, M.; ALSHAMMA'A, A. Embedded Smart Antenna for Non-Destructive Testing and Evaluation (NDT\&E) of Moisture Content and Deterioration in Concrete. Sensors, v. 19, 547, 2019.

TRAN, Q. H.; HUH, J.; KANG, C.; LEE, B. Y.; KIM, I.-T.; AHN, J.-H. Detectability of Subsurface Defects with Different Width-to-Depth Ratios in Concrete Structures Using Pulsed Thermography. Journal of Nondestructive Evaluation, v. 37, 2018.

ULLAH, S.; JEONG, M.; LEE, W. Nondestructive Inspection of Reinforced Concrete Utility Poles with ISOMAP and Random Forest. Sensors, v.18, 3463, 2018.

VASHPANOV, Y.; SON, J-Y; HEO, G.; PODOUSOVA, T.; KIM, Y.S. Determination of Geometric Parameters of Cracks in Concrete by Image Processing. Advances in Civil Engineering, vol. 2019, p.1-14, 2019.

WU, J.; XU, C.; QI, B.; HERNANDEZ, F.C.R. Detection of Impact Damage on PVA-ECC Beam Using Infrared Thermography. Applied Sciences, v. 8, 839, 2018.

YE, J.; KOBAYASHI, T.; IWATA, M.; TSUDA, H.; MURAKAWA, M. Computerized Hammer Sounding Interpretation for Concrete Assessment with Online Machine Learning. Sensors, v.18, 833, 2018.

ZHANG, Y.; LAROSE, E.; MOREAU, L.; D’OZOUVILLE, G. Three-dimensional in-situ imaging of cracks in concrete using diffuse ultrasound. Structural Health Monitoring, v. 17, p. 279-284, 2017.

ZHOU, F.; CHEN, Z.; LIU, H.; CUI, J.; SPENSER, B.F.; FANG, G. Simultaneous Estimation of Rebar Diameter and Cover Thickness by a GPR-EMI Dual Sensor. Sensors, v.18, 2969, 2018. 